\title{
DIVISIBILITY PROPERTIES OF THE $q$-TANGENT NUMBERS
}

\author{
GEORGE E. ANDREWS ${ }^{1}$ AND IRA GESSEL
}

\begin{abstract}
The $q$-tangent number $T_{2 n+1}(q)$ is shown to be divisible by $(1+q)\left(1+q^{2}\right) \cdots\left(1+q^{n}\right)$. Related divisibility questions are discussed.
\end{abstract}

1. Introduction. The tangent numbers $T_{2 n+1}$ are integers defined by

$$
\sum_{n=0}^{\infty} \frac{T_{2 n+1} x^{n}}{(2 n+1) !}=\tan x
$$

Numerous properties of the tangent number are known; in particular [2, p. 259]:

$$
T_{2 n+1}=4^{n+1}\left|G_{2 n+2}\right| /(n+1),
$$

where $G_{n}$ is an integer called the Genocchi number. Thus it is clear from (1.2) that $T_{2 n+1}$ is always divisible by a high power of 2 .

A natural $q$-analog of the tangent numbers is given by

$$
\begin{aligned}
\sum_{n=0}^{\infty} \frac{T_{2 n+1}(q) x^{n}}{(q)_{2 n+1}} & =\frac{\sin _{q} x}{\cos _{q} x} \\
& =\sum_{n=0}^{\infty} \frac{(-1)^{n} x^{2 n+1}}{(q)_{2 n+1}} / \sum_{n=0}^{\infty} \frac{(-1)^{n} x^{2 n}}{(q)_{2 n}}
\end{aligned}
$$

where $(A)_{n}=(A ; q)_{n}=(1-A)(1-A q) \cdots\left(1-A q^{n-1}\right) ;$ R. P. Stanley [4] has given a combinatorial interpretation of the polynomials $T_{2 n+1}(q)$ which shows that all the coefficients are nonnegative.

One of us [3] has shown that $T_{2 n+1}(q)$ is divisible by the cyclotomic polynomials $\phi_{2}(q), \phi_{4}(q), \ldots, \phi_{2 n}(q)$ through a study of properties of Gaussian polynomials in cyclotomic fields. Our object here is to derive the following result on $q$-tangent numbers which is analogous to the fact that $T_{2 n+1}$ is divisible by a high power of 2 :

THEOREM 1. The polynomial $T_{2 n+1}(q)$ is divisible by $(1+q)\left(1+q^{2}\right) \cdots(1$ $\left.+q^{n}\right)$.

We conclude with a few comments about other divisibility properties of $T_{2 n+1}(q)$ that are derivable using our method. The assertion in Theorem 1 was a conjecture made by $M$. P. Schützenberger at the combinatorics

Received by the editors June 9, 1977.

AMS (MOS) subject classifications (1970). Primary 05A15, 05A19; Secondary 33A30.

'Partially supported by the National Science Foundation Grant MSP 74-07282.

c) American Mathematical Society 1978 
conference at Oberwohlfach in February, 1975.

2. Proof of Theorem 1. To prove this result we require two lemmas.

LEMMA 1. For nonnegative integers $N$ and $j$, the expression

$$
\left[\begin{array}{c}
2 N+1 \\
2 j
\end{array}\right] \frac{(1+q)\left(1+q^{2}\right) \cdots\left(1+q^{j}\right)}{\left(1+q^{N}\right)\left(1+q^{N-1}\right) \cdots\left(1+q^{N-j+1}\right)}
$$

is a polynomial in $q$, where $\left[{ }_{M}^{N}\right]$ is the Gaussian polynomial

$$
\left[\begin{array}{l}
N \\
M
\end{array}\right]=\frac{(q)_{N}}{(q)_{M}(q)_{N-M}}
$$

Proof. Obviously the expression in question is a rational function and the roots of the denominator are roots of unity. To prove Lemma 1 we need only show that each zero of the denominator appears with at least as large multiplicity in the numerator as in the denominator.

Now if $\rho$ is a primitive $k$ th root of unity then $\rho$ is a simple root of $1-q^{M}$ if and only if $k \mid M$. Furthermore we know a priori (due to the recurrences for Gaussian polynomials) that $\left[\begin{array}{c}2 N+1 \\ 2 j\end{array}\right]$ is a polynomial. Consequently for each integer $l$ with $1 \leqslant l \leqslant 2 j$, we see that $l$ must divide at least $[2 j / l]$ of the numbers $2 N+1,2 N, 2 N-1, \ldots, 2 N-2 j+2$ (otherwise this Gaussian polynomial would not be a polynomial). Now

$$
\begin{aligned}
& {\left[\begin{array}{rr}
2 N+1 & \\
& 2 j
\end{array}\right] \frac{(1+q)\left(1+q^{2}\right) \cdots\left(1+q^{j}\right)}{\left(1+q^{N}\right)\left(1+q^{N-1}\right) \cdots\left(1+q^{N-j+1}\right)}} \\
& \quad=\frac{\left(1-q^{2 N+1}\right)\left(1-q^{N}\right)\left(1-q^{2 N-1}\right)\left(1-q^{N-1}\right) \cdots\left(1-q^{2 N-2 j+3}\right)\left(1-q^{N-j+1}\right)}{\left(1-q^{j}\right)\left(1-q^{2 j-1}\right)\left(1-q^{j-1}\right)\left(1-q^{2 j-3}\right) \cdots(1-q)(1-q)}
\end{aligned}
$$

and one sees that this is the same as the expression for $\left[{ }_{2 j}^{2 N+1}\right]$ except that each even exponent in numerator and denominator has been divided by 2 . Thus the divisibility properties previously described are preserved since the only change is that $j$ numerator exponents and $j$ denominator exponents have been divided by 2 which of course does not affect whether a denominator exponent divides a numerator exponent (i.e. if $l$ is odd and $l \mid 2 M$ then $l \mid M$, if $l$ is even and $l \mid 2 M$ then $\left.\frac{l}{2} \mid M\right)$. Thus the denominator of

$$
\left[\begin{array}{c}
2 N+1 \\
2 j
\end{array}\right] \frac{(1+q)\left(1+q^{2}\right) \cdots\left(1+q^{j}\right)}{\left(1+q^{N}\right)\left(1+q^{N-1}\right) \cdots\left(1+q^{N-j+1}\right)}
$$

has no zeros that are not cancelled by those of the numerator. This proves Lemma 1.

LEMMA 2. The q-tangent numbers satisfy 


$$
T_{2 N+1}(q)+\sum_{j=1}^{N}(-q)_{2 j-1}\left[\begin{array}{c}
2 N+1 \\
2 j
\end{array}\right](-1)^{j} T_{2 N+1-2 j}(q)
$$

$$
=(-1)^{N}(-q)_{2 N}
$$

where $(a)_{n}=(a ; q)_{n}=(1-a)(1-a q) \cdots\left(1-a q^{n-1}\right),(a)_{0}=1$.

Proof. We have

$$
\sum_{n=0}^{\infty} \frac{T_{2 n+1}(q) x^{2 n+1}}{(q)_{2 n+1}}=\sum_{n=0}^{\infty} \frac{(-1)^{n} x^{2 n+1}}{(q)_{2 n+1}} / \sum_{n=0}^{\infty} \frac{(-1)^{n} x^{2 n}}{(q)_{2 n}} .
$$

Now (here $i=\sqrt{-1}$ )

$$
\begin{gathered}
\sum_{n=0}^{\infty} \frac{(-1)^{n} x^{2 n+1}}{(q)_{2 n+1}}=\sum_{n=0}^{\infty} \frac{i^{n-1} x^{n}}{(q)_{n}} \frac{\left(1-(-1)^{n}\right)}{2} \\
=\frac{1}{2 i}\left(\frac{1}{(i x)_{\infty}}-\frac{1}{(-i x)_{\infty}}\right) \quad(\text { by }[1, \text { p. 19, equation (2.2.5)]); } \\
\sum_{n=0}^{\infty} \frac{(-1)^{n} x^{2 n}}{(q)_{2 n}}=\sum_{n=0}^{\infty} \frac{i^{n} x^{n}}{(q)_{n}} \frac{\left(1+(-1)^{n}\right)}{2} \\
=\frac{1}{2}\left(\frac{1}{(i x)_{\infty}}+\frac{1}{(-i x)_{\infty}}\right)
\end{gathered}
$$

Therefore

$$
\begin{aligned}
\sum_{n=0}^{\infty} \frac{T_{2 n+1}(q) x^{2 n+1}}{(q)_{2 n+1}} & =\frac{1}{2 i}\left(\frac{1}{(i x)_{\infty}}-\frac{1}{(-i x)_{\infty}}\right) / \frac{1}{2}\left(\frac{1}{(i x)_{\infty}}+\frac{1}{(-i x)_{\infty}}\right) \\
& =\frac{1}{i} \frac{(-i x)_{\infty}-(i x)_{\infty}}{(-i x)_{\infty}+(i x)_{\infty}}=\frac{1}{i} \frac{(-i x)_{\infty} /(i x)_{\infty}-1}{(-i x)_{\infty} /(i x)_{\infty}+1}
\end{aligned}
$$

Clearing the denominator on the right and utilizing the $q$-binomial series

$$
\sum \frac{(A)_{n} z^{n}}{(q)_{n}}=\frac{(A z)_{\infty}}{(z)_{\infty}}
$$

[1, p. 17, equation (2.2.1)], we find that

$$
\left(1+\sum_{n=0}^{\infty} \frac{(-1)_{n}(i x)^{n}}{(q)_{n}}\right) \sum_{n=0}^{\infty} \frac{T_{2 n+1}(q) x^{2 n+1}}{(q)_{2 n+1}}=\frac{1}{i} \sum_{n=1}^{\infty} \frac{(-1)_{n}(i x)^{n}}{(q)_{n}}
$$

Let us now compare the real parts of the coefficient of $x^{2 N+1}$ in this last identity:

$$
2 T_{2 N+1}(q)+\sum_{j=1}^{N}(-1)_{2 j}\left[\begin{array}{c}
2 N+1 \\
2 j
\end{array}\right](-1)^{j} T_{2 N+1-2 j}(q)=(-1)_{2 N+1}(-1)^{N},
$$

and if we divide each side of this identity by 2 we obtain the result stated in Lemma 2. 
THEOREM 1. The polynomial $(1+q)\left(1+q^{2}\right) \cdots\left(1+q^{N}\right)$ divides the polynomial $T_{2 N+1}(q)$.

Proof. The result is immediate for $N=0,1$ since $T_{1}=1$ and $T_{3}=q(1+$ $q)$. Let us now assume the result true up to but not including $N$.

Now

$$
\begin{aligned}
(-q)_{2 j-1}\left[\begin{array}{c}
2 N+1 \\
2 j
\end{array}\right] & =(-q)_{j}\left(-q^{j+1}\right)_{j-1}\left[\begin{array}{c}
2 N+1 \\
2 j
\end{array}\right] \\
= & \left(1+q^{N}\right)\left(1+q^{N-1}\right) \cdots\left(1+q^{N-j+1}\right)\left(-q^{j+1}\right)_{j-1} \\
& \times \frac{(1+q)\left(1+q^{2}\right) \cdots\left(1+q^{j}\right)}{\left(1+q^{N}\right)\left(1+q^{N-1}\right) \cdots\left(1+q^{N-j+1}\right)}\left[\begin{array}{c}
2 N+1 \\
2 j
\end{array}\right] .
\end{aligned}
$$

Hence by Lemma $1,\left(1+q^{N}\right)\left(1+q^{N-1}\right) \cdots\left(1-q^{N-j+1}\right)$ is factor of the polynomial $(-q)_{2 j-1}\left[\begin{array}{c}2 N+1 \\ 2 j\end{array}\right]$. By the induction hypothesis $(1+q)(1+$ $\left.q^{2}\right) \cdots\left(1+q^{N-j}\right)$ is a factor of $T_{2 N+1-2 j}(q)$. Hence for $1 \leqslant j \leqslant N$, we see that $(-q)_{N}$ is a factor of

$$
(-1)^{j}(-q)_{2 j-1}\left[\begin{array}{c}
2 N+1 \\
2 j
\end{array}\right] T_{2 N+1-2 j}(q),
$$

and since $(-q)_{N}$ is obviously a factor of $(-q)_{2 N}$ we deduce from Lemma 2 that $(-q)_{N}$ is a factor of $T_{2 N+1}(q)$ as well. Thus Theorem 1 follows by induction.

3. Conclusion. First we note that the result mentioned in the Introduction about the divisibility of the $T_{2 n+1}(q)$ by the cyclotomic polynomials $\phi_{2}(q)$, $\phi_{4}(q), \ldots, \phi_{2 n}(q)$ now follows from Theorem 1 since $\phi_{2 n}(q)$ divides $\left(1+q^{n}\right)$.

We also note that the divisibility of $T_{2 n+1}(q)$ by specific factors of the form $1+q^{j}$ can be handled again by Lemma 2 . For example:

THEOREM 2. The polynomial $(1+q)^{n}$ is a factor of the q-tangent number $T_{2 n+1}(q)$.

Proof. The result is obvious for $n=0,1$ since $T_{1}(q)=1$ and $T_{3}(q)=q(1$ $+q)$. Assume the theorem true up to but not including $n$. Now since $1+q^{2 M+1}=(1+q)\left(1-q+q^{2}-\cdots+q^{2 M}\right)$, we see that $(1+q)^{j}$ is a factor of $(-q)_{2 j-1}$. By the induction hypothesis $(1+q)^{N-j}$ is a factor of $T_{2 N+1-2 j}(q)$. Hence $(1+q)^{N}$ is a factor of

$$
(-q)_{2 j-1}\left[\begin{array}{c}
2 N+1 \\
2 j
\end{array}\right](-1)^{j} T_{2 N+1-2 j}(q)
$$

and since $(1+q)^{N}$ is also a factor of $(-q)_{2 N}$, we deduce from Lemma 2 that $(1+q)^{N}$ is a factor of $T_{2 N+1}(q)$.

\section{REFERENCES}

1. G. E. Andrews, The theory of partitions, Encyclopedia of Mathematics and its Applications, Vol. 2, Addison-Wesley, Reading, Mass., 1976.

2. L. Comtet, Advanced combinatorics, Riedel, Dordrecht, 1974. 
3. I. Gessel, Exponential generating functions $(\bmod p)$ and their q-analogs (in prep.).

4. R. P. Stanley, Binomial posets, Möbius inversion, and permutation enumeration, J. Combinatorial Theory Ser. A 20 (1976), 336-356.

Department of Mathematics, Pennsylvania State University, University Park, PennSYlvanin 16802 (Current address of G. E. Andrews)

Department of Mathematics, Massachusetts Institute of Technology, Cambridge, MASSACHUSETTS 02139

Current address (Ira Gessel): IBM T. J. Watson Research Center, Yorktown Heights, New York 10598 